\title{
GLOBAL DYNAMICS OF THE KUMMER-SCHWARZ DIFFERENTIAL EQUATION
}

\author{
JAUME LLIBRE ${ }^{1}$ AND CLAUDIO VIDAL ${ }^{2}$
}

\begin{abstract}
This paper studies the Kummer-Schwarz differential equation $2 \dot{x} \dddot{x}-3 \ddot{x}^{2}=0$ which is of special interest due to its relationship with the Schwarzian derivative. This differential equation is transformed into a first order differential system in $\mathbb{R}^{3}$, and we provide a complete description of its global dynamics adding the infinity.
\end{abstract}

\section{INTRODUCTION AND STATEMENTS OF MAIN RESULTS}

The Schwarzian derivative

$$
\{x, t\}=\frac{\dddot{x}(t)}{\dot{x}(t)}-\frac{3}{2}\left(\frac{\ddot{x}(t)}{\dot{x}(t)}\right)^{2},
$$

plays an important role in the treatment of univalent functions, see details in [5] and references therein. Here the dot denotes derivative with respect to the independent variable $t$. When the right hand in equation (1) is taken at zero, the resulting equation is the KummerSchwarz equation which is given by

$$
2 \dot{x} \dddot{x}-3 \ddot{x}^{2}=0,
$$

and is of special interest due to its relationship to the Schwarzian derivative and its exceptional algebraic properties. This equation is also encountered in the study of geodesic curves in spaces of constant curvature, Lie lists the characteristic functions for its contact symmetries, see more results on this differential equation in [1], [4], [5] and [6]. But up to now nobody has described its global dynamics. This will be the objective of this paper.

The Kummer-Schwarz equation of third order (2) can be written as the following rational differential system of first order

$$
\dot{x}=y, \quad \dot{y}=z, \quad \dot{z}=\frac{3 z^{2}}{2 y},
$$

2010 Mathematics Subject Classification. Primary 34C10, 34C25.

Key words and phrases. Kummer-Schwarz equation, global dynamics, integrability, Poincaré compactification. 
in $\mathbb{R}^{3}$. Rescaling the time according to $d \tau / d t=2 y$, we obtain the equivalent polynomial differential system (outside the plane $y=0$ )

$$
x^{\prime}=2 y^{2}, \quad y^{\prime}=2 y z, \quad z^{\prime}=3 z^{2},
$$

here the prime denotes derivative with respect to the new independent variable $\tau$. This differential system is called the Kummer-Schwarz differential system in $\mathbb{R}^{3}$.

We will study the flow of the polynomial differential system (4) in the phase space $\mathbb{R}^{3}$, of course, in order to describe the flow of the differential system (3) we must omit the plane $y=0$.

\subsection{Symmetries and reduction of the flow to the quadrant} $y \geq 0$ and $z \geq 0$. The differential system (4) is invariant under the following two symmetries

$$
S_{1}(x, y, z)=(x,-y, z), \quad \text { and } \quad S_{2}(x, y, z, \tau)=(-x, y,-z,-\tau) .
$$

The symmetry $S_{1}$ says that the flow of system (4) is symmetric with respect to the plane $y=0$. Therefore, if $(x(\tau), y(\tau), z(\tau))$ is a solution of (4), then $(x(\tau),-y(\tau), z(\tau))$ is also solution of (4). The symmetry $S_{2}$ says that the flow of system (4) is symmetric with respect to the $y$-axis reversing the sense of the orbits. Therefore, if $(x(\tau), y(\tau), z(\tau))$ is a solution of $(4)$, then $(-x(-\tau), y(\tau),-z(-\tau))$ is also solution of $(4)$. Using both symmetries in order to describe the flow of system (4) in $\mathbb{R}^{3}$ it is enough to describe the flow of system (4) on the quadrant

$$
Q=\left\{(x, y, z) \in \mathbb{R}^{3}: y \geq 0, z \geq 0\right\} .
$$

1.2. The Poincaré compactification. It is know that a polynomial differential system in $\mathbb{R}^{3}$ can be extended to a unique analytic differential system on the closed ball $B$ of radius 1 centered at the origin of $\mathbb{R}^{3}$, called the Poincaré ball. More precisely, the whole space $\mathbb{R}^{3}$ is identified with the interior of $B$, and the infinity of $\mathbb{R}^{3}$ is identified with the boundary of $B$, i.e. with the 2 -dimensional sphere $\mathbb{S}^{2}$. For more details see [2] and [7], and the appendix of this paper. The know technique for making such an extension is called the Poincaré compactification and it allows to study the dynamics of a polynomial differential system in a neighborhood of infinity. Poincaré introduced this compactification for polynomial differential systems in $\mathbb{R}^{2}$.

1.3. The global dynamics. Our main result is the description of the global dynamics of the Kummer-Schwarz differential system (4) on the compactified quadrat $\bar{Q}$ of $Q$ inside the Poincaré ball $B$, see Figure 1. More precisely, we describe all the $\alpha$ - and $\omega$-limit sets of all the orbits of the Kummer-Schwarz differential system (4). For the standard 
definitions of orbit, $\alpha$ - and $\omega$-limit sets of an orbit, and of the Poincaré compactification, see for instance [3].

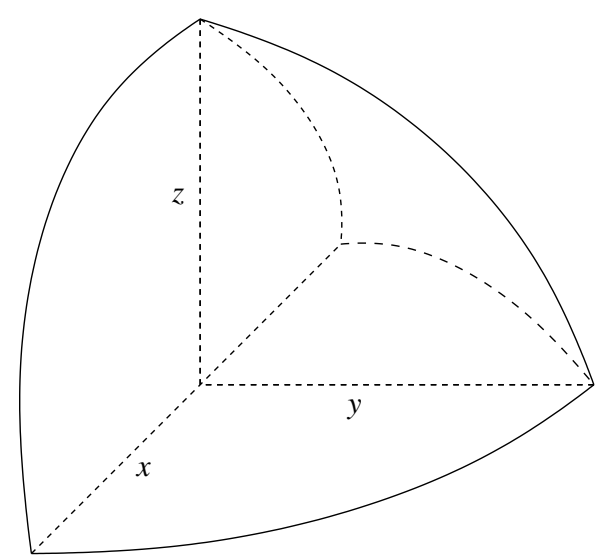

Figure 1. The quadrant $\bar{Q}$ of the Poincaré ball $B$.

Theorem 1. The following statements hold for the Kummer-Schwarz differential system (4).

(a) On the quadrant $\bar{Q}$ the equilibrium points are all the points of the $x$-axis, including its endpoints at infinity ( $X_{-}$at the end of the negative $x$-axis and $X_{+}$at the end of the positive $x$-axis), and additionally the endpoint $Z_{+}$at infinity of the positive $z_{-}^{-}$ axis, see Figure 2.

(b) On the invariant boundary $y=0$ of the quadrant $\bar{Q}$ the orbits are the half-straight lines parallel to the $z$-axis having $\alpha$-limit an equilibrium point of the $x$-axis and $\omega$-limit the equilibrium point $Z_{+}$, see Figure 2.

(c) On the invariant boundary $z=0$ of the quadrant $\bar{Q}$ the orbits are the straight lines parallel to the $x$-axis having $\alpha$-limit the equilibrium point $X_{-}$and $\omega$-limit the equilibrium point $X_{+}$, see Figure 2.

(d) On the infinity $\mathbb{S}^{2} \cap \bar{Q}$ the flow is qualitatively the one described in Figure 3, i.e. without taken into account the three equilibrium points $Z_{+}, X_{-}$and $X_{+}$at the infinity of the quadrant $\bar{Q}$ all the other orbits have $\omega$-limit at the equilibrium point $Z_{+}$, and $\alpha_{-}$ limit either at $X_{-}$, or at $X_{+}$. 
(e) The explicit solution $(x(\tau), y(\tau), z(\tau))$ of the differential system (4) such that $(x(0), y(0), z(0))=\left(x_{0}, y_{0}, z_{0}\right)$ is

$$
\begin{aligned}
& x(\tau)=x_{0}+\frac{2 y_{0}^{2}}{z_{0}}\left(\frac{1}{\left(1-3 z_{0} \tau\right)^{1 / 3}}-1\right), \\
& y(\tau)=\frac{y_{0}}{\left(1-3 z_{0} \tau\right)^{2 / 3}} \\
& z(\tau)=\frac{z_{0}}{1-3 z_{0} \tau}
\end{aligned}
$$

(f) Let $\gamma$ be an orbit contained in the interior of the quadrant $\bar{Q}$. Then the $\alpha$-limit of $\gamma$ is the equilibrium point $X_{-}$and its $\omega-$ limit is the equilibrium point $Z_{+}$.

(g) The differential system (4) has two independent first integrals $H_{1}=z^{2} / y^{3}$ and $H_{2}=x-2 y^{2} / z$. The set $\left\{H_{1}=h_{1}\right\} \cap\left\{H_{2}=\right.$ $\left.h_{2}\right\} \cap Q$ is an orbit $\gamma$ with endpoints at $X_{-}$and $Z_{+}$.

The proof of Theorem 1 is given in section 2 .

The two independent first integrals $H_{1}$ and $H_{2}$ of statement (g) of Theorem 1 are due to Goviender and Leach [4].

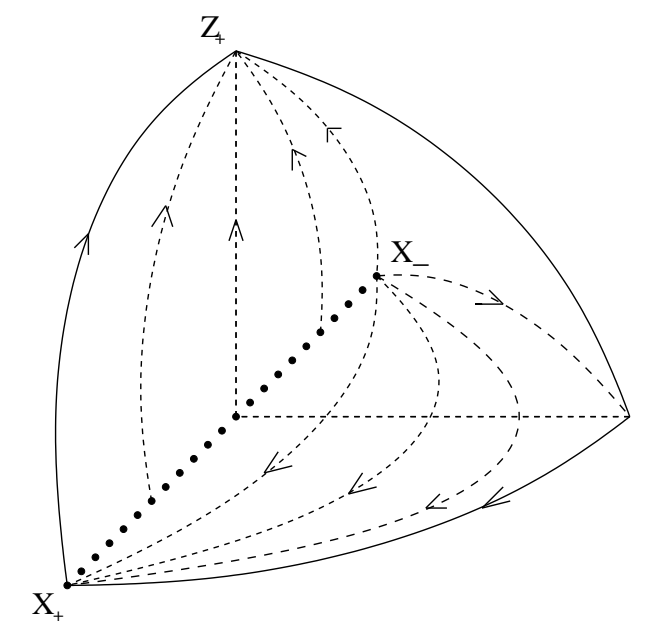

Figure 2. The flow on the invariant planes $y=0$ and $z=0$ restricted to the quadrant $\bar{Q}$.

\section{Proof of Theorem 1}

This section is dedicated to prove the different statements of Theorem 1. 


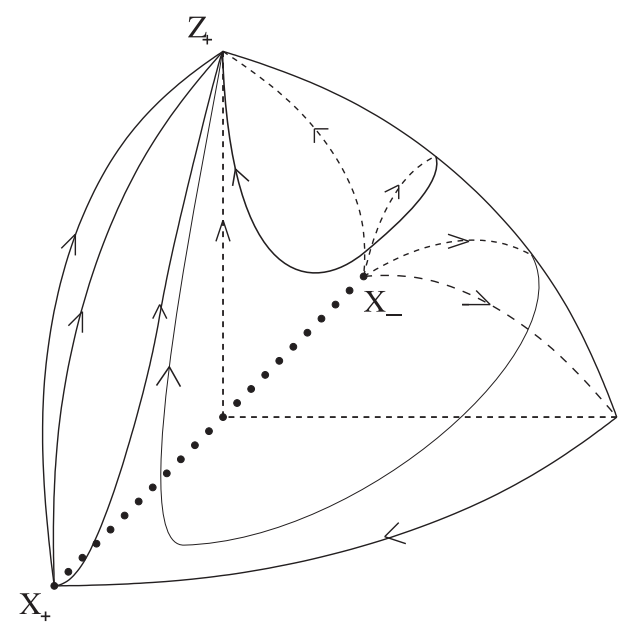

Figure 3. The flow on the infinity restricted to the quadrant $\bar{Q}$.

Proof of statement (a) of Theorem 1. From the equations of the differential system (4) it follows immediately that the $x$-axis is filled with equilibrium points, because $x^{\prime}=y^{\prime}=z^{\prime}=0$ when $y=z=0$. Now we shall study the equilibriums points at the infinity of the quadrant $\bar{Q}$ using the Poincaré compactification of $\mathbb{R}^{3}$ described in the appendix.

We start studying the equilibrium points at the infinity located on the local chart $U_{1}$, i.e. in $x>0$ and its boundary at infinity. Thus the compactified differential system (4) in the local chart $U_{1}$ is given by

$$
\dot{z}_{1}=-2 z_{1}^{3}+2 z_{1} z_{2}, \quad \dot{z}_{2}=-2 z_{1}^{2} z_{2}+3 z_{2}^{2}, \quad \dot{z}_{3}=-2 z_{1}^{2} z_{3} .
$$

At the infinity $z_{3}=0$ of $U_{1}$, i.e. in the points of the sphere $\mathbb{S}^{2}$ system (6) reduces to

$$
\dot{z}_{1}=2 z_{1}\left(-z_{1}^{2}+z_{2}\right), \quad \dot{z}_{2}=z_{2}\left(-2 z_{1}^{2}+3 z_{2}\right) .
$$

So, the unique equilibrium point at the infinity of $U_{1}$ is the origin $(0,0,0)$ of $U_{1}$. Its linear part has all its eigenvalues equal to zero. Therefore we need to study it using the technique of blow ups, see for more details the Chapter 3 of [3]. Then, we obtain that the local phase portrait of the equilibrium point $(0,0)$ of the differential system $(7)$ is qualitatively the one of Figure 4 . The equilibrium point $(0,0,0)$ of $U_{1}$ corresponds to the endpoint $X_{+}$of the positive $x$-axis.

The local phase portrait at the equilibrium point $(0,0,0)$ of $V_{1}$ which corresponds to the endpoint $X_{-}$of the negative $x$-axis, is obtained doing symmetry with respect to the center of the sphere $\mathbb{S}^{2}$ and reversing 


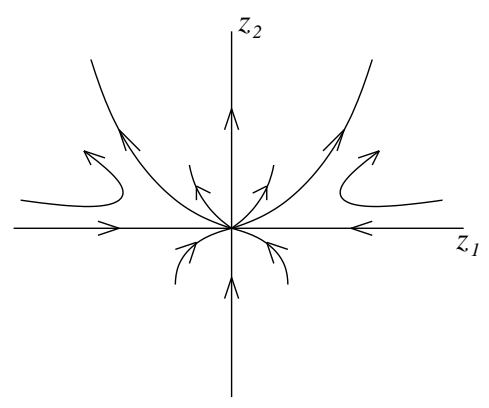

Figure 4. The local phase portrait of the equilibrium point $(0,0)$ of the differential system $(7)$.

the orientation of the orbits because the degree of the polynomial differential system (4) is 2 .

The flow of system (4) in the local chart $U_{2}$ is given by the differential system

$$
\dot{z}_{1}=-2 z_{1} z_{2}+2, \quad \dot{z}_{2}=-2 z_{1}^{2} z_{2}+3 z_{2}^{2}, \quad \dot{z}_{3}=-2 z_{2} z_{3} .
$$

So there are no equilibrium points at infinity in this local chart.

In the local chart $U_{3}$ the system (4) becomes

$$
\dot{z}_{1}=-3 z_{1}+2 z_{2}^{2}, \quad \dot{z}_{2}=-z_{2}, \quad \dot{z}_{3}=-3 z_{3} .
$$

At the infinity of $U_{3}$ the point $(0,0,0)$ is the unique equilibrium point, and its linear part has the eigenvalues -1 and -3 with multiplicity two. Therefore, by the Hartman Theorem this equilibrium point is a local attractor, and it corresponds to the endpoint $Z_{+}$of the positive $z$-axis.

Proof of statement (b) of Theorem 1. From the differential system (4) it follows that $x^{\prime}=0$ and $y^{\prime}=0$ when $y=0$, so the plane $y=0$ and the straight lines $\{y=0\} \cap\{x=$ constant $\}$ are invariant by the flow of system (4). In other words, if an orbit of the differential system (4) has a point in $y=0$, or in $\{y=0\} \cap\{x=$ constant $\}$ then the whole orbit is contained in that plane or straight line.

In short, on the invariant boundary $y=0$ of the quadrant $\bar{Q}$ the orbits are the half-straight lines parallel to the $z$-axis having $\alpha$-limit an equilibrium point of the $x$-axis and $\omega$-limit the equilibrium point $Z_{+}$, see for more details the proof of statement (a).

Proof of statement (c) of Theorem 1. From the differential system (4) we have that $y^{\prime}=0$ and $z^{\prime}=0$ when $z=0$, so the plane $z=0$ and 
the straight lines $\{z=0\} \cap\{y=$ constant $\}$ are invariant by the flow of system (4).

In summary, on the invariant boundary $z=0$ of the quadrant $\bar{Q}$ the orbits are the straight lines parallel to the $x$-axis having $\alpha$-limit the equilibrium point $X_{-}$and $\omega$-limit the equilibrium point $X_{+}$, see again for more details the proof of statement (a).

Proof of statement $(d)$ of Theorem 1. From the fact that the infinity $\mathbb{S}^{2}$ is invariant by the compactified flow of the polynomial differential system (4), and the local phase portraits at the three equilibrium points $Z_{+}, X_{-}$and $X_{+}$at the infinity of the quadrant $\bar{Q}$ studied in the proof of statement (a), it follows that on the infinity $\mathbb{S}^{2} \cap \bar{Q}$ the flow is qualitatively the one described in Figure 3.

Proof of statement (e) of Theorem 1. Let $(x(\tau), y(\tau), z(\tau))$ be the solution of the differential system (4) such that $(x(0), y(0), z(0))=\left(x_{0}, y_{0}\right.$, $\left.z_{0}\right)$. From the differential equation $z^{\prime}=3 z^{2}$ it follows easily that $z(\tau)=z_{0} /\left(1-3 z_{0} \tau\right)$. Substituting $z=z(\tau)$ in $y^{\prime}=2 y z$ we obtain that $y(\tau)=y_{0} /\left(1-3 z_{0} \tau\right)^{2 / 3}$. Finally, substituting $y=y(\tau)$ in $x^{\prime}=2 y^{2}$ and integrating we get that

$$
x(\tau)=x_{0}+\frac{2 y_{0}^{2}}{z_{0}}\left(\frac{1}{\left(1-3 z_{0} \tau\right)^{1 / 3}}-1\right) .
$$

Proof of statement $(f)$ of Theorem 1. Since $x^{\prime}=2 y^{2}>0$ and $z^{\prime}=$ $3 z^{2}>0$ in the interior of the quadrant $\bar{Q}$, for every orbit $\gamma$ contained in the interior of the quadrant $\bar{Q}$ we have that its $\alpha$-limit has its $x-$ coordinate equal to $-\infty$ and its $z$-coordinate equal to 0 , and its $\omega$-limit has its $x$-coordinate equal to $+\infty$ and its $z$-coordinate equal to $+\infty$. Taking into account either the solution of statement (e), or the phase portrait on the boundary of the quadrant $\bar{Q}$ described in the statements (b), (c) and (d) we get that the $\alpha$-limit of $\gamma$ is the equilibrium point $X_{-}$and its $\omega$-limit is the equilibrium point $Z_{+}$.

Proof of statement $(g)$ of Theorem 1. Let $H_{1}=z^{2} / y^{3}$ and $H_{2}=x-$ $2 y^{2} / z$. Then, since

$$
\frac{d H_{k}}{d \tau}=\frac{\partial H_{k}}{\partial x} x^{\prime}+\frac{\partial H_{k}}{\partial y} y^{\prime}+\frac{\partial H_{k}}{\partial z} z^{\prime}=0
$$

for $k=1,2$, we obtain that $H_{1}$ and $H_{2}$ are first integrals of the differential system (4), i.e. they are constant on the solutions of system (4) where they are defined. Since there gradients are linearly independent 
except at the points of $z=0$ and $y=0$ which have zero Lebesgue measure, these two first integrals are independent.

It is not difficult to show that the set $\left\{H_{1}=h_{1}\right\} \cap\left\{H_{2}=h_{2}\right\} \cap Q$ has a unique component. Then, from either statement (e), or statement (f) it follows that this set is formed by an orbit $\gamma$ with endpoints at $X_{-}$and $Z_{+}$.

\section{The appendix: Poincaré compactification of $\mathbb{R}^{3}$}

In $\mathbb{R}^{3}$ we consider the polynomial differential system

$$
\dot{x}=P^{1}(x, y, z), \quad \dot{y}=P^{2}(x, y, z), \quad \dot{z}=P^{3}(x, y, z),
$$

or equivalently its associated polynomial vector field $X=\left(P^{1}, P^{2}, P^{3}\right)$. The degree $n$ of $X$ is defined as $n=\max \left\{\operatorname{deg}\left(P^{i}\right): i=1,2,3\right\}$.

Let $\mathbb{S}^{3}=\left\{y=\left(y_{1}, y_{2}, y_{3}, y_{4}\right) \in \mathbb{R}^{4}:\|y\|=1\right\}$ be the unit sphere in $\mathbb{R}^{4}$, and

$$
\mathbb{S}_{+}=\left\{y \in \mathbb{S}^{3}: y_{4}>0\right\} \quad \text { and } \mathbb{S}_{-}=\left\{y \in \mathbb{S}^{3}: y_{4}<0\right\}
$$

be the northern and southern hemispheres, respectively. The tangent space to $\mathbb{S}^{3}$ at the point $y$ is denoted by $T_{y} \mathbb{S}^{3}$. Then, the tangent hyperplane

$$
T_{(0,0,0,1)} \mathbb{S}^{3}=\left\{\left(x_{1}, x_{2}, x_{3}, 1\right) \in \mathbb{R}^{4}:\left(x_{1}, x_{2}, x_{3}\right) \in \mathbb{R}^{3}\right\}
$$

is identified with $\mathbb{R}^{3}$.

We consider the central projections

$$
f_{+}: \mathbb{R}^{3}=T_{(0,0,0,1)} \mathbb{S}^{3} \rightarrow \mathbb{S}_{+} \quad \text { and } \quad f_{-}: \mathbb{R}^{3}=T_{(0,0,0,1)} \mathbb{S}^{3} \rightarrow \mathbb{S}_{-},
$$

defined by

$$
f_{+}(x)=\frac{1}{\Delta x}\left(x_{1}, x_{2}, x_{3}, 1\right) \text { and } f_{-}(x)=-\frac{1}{\Delta x}\left(x_{1}, x_{2}, x_{3}, 1\right),
$$

where $\Delta x=\left(1+\sum_{i=1}^{3} x_{i}^{2}\right)^{1 / 2}$. Through these central projections, $\mathbb{R}^{3}$ can be identified with the northern and the southern hemispheres, respectively. The equator of $\mathbb{S}^{3}$ is $\mathbb{S}^{2}=\left\{y \in \mathbb{S}^{3}: y_{4}=0\right\}$. Clearly, $\mathbb{S}^{2}$ can be identified with the infinity of $\mathbb{R}^{3}$.

The maps $f_{+}$and $f_{-}$define two copies of $X$, one $D f_{+} \circ X$ in the northern hemisphere and the other $D f_{-} \circ X$ in the southern one. Denote by $\bar{X}$ the vector field on $\mathbb{S}^{3} \backslash \mathbb{S}^{2}=\mathbb{S}_{+} \cup \mathbb{S}_{-}$which restricted to $\mathbb{S}_{+}$ coincides with $D f_{+} \circ X$ and restricted to $\mathbb{S}_{-}$coincides with $D f_{-} \circ X$.

In what follows we shall work with the orthogonal projection of the closed northern hemisphere to $y_{4}=0$. Note that this projection is a closed ball $B$ of radius one, whose interior is diffeomorphic to $\mathbb{R}^{3}$ and whose boundary $\mathbb{S}^{2}$ corresponds to the infinity of $\mathbb{R}^{3}$. We shall extend 
analytically the polynomial vector field $\bar{X}$ to the boundary, in such a way that the flow on the boundary is invariant. This new vector field on $B$ will be called the Poincaré compactification of $X$, and $B$ will be called the Poincaré ball. Poincaré introduced this compactification for polynomial vector fields in $\mathbb{R}^{2}$, and its extension to $\mathbb{R}^{m}$ can be found in [7].

The expression for $\bar{X}(y)$ on $\mathbb{S}_{+} \cup \mathbb{S}_{-}$is

$$
\bar{X}(y)=y_{4}\left(\begin{array}{ccc}
1-y_{1}^{2} & -y_{2} y_{1} & -y_{3} y_{1} \\
-y_{1} y_{2} & 1-y_{2}^{2} & -y_{3} y_{2} \\
-y_{1} y_{3} & -y_{2} y_{3} & 1-y_{3}^{2} \\
-y_{1} y_{4} & -y_{2} y_{4} & -y_{3} y_{4}
\end{array}\right)\left(\begin{array}{l}
P^{1} \\
P^{2} \\
P^{3}
\end{array}\right)
$$

where $P^{i}=P^{i}\left(y_{1} /\left|y_{4}\right|, y_{2} /\left|y_{4}\right|, y_{3} /\left|y_{4}\right|\right)$. Written in this way $\bar{X}(y)$ is a vector field in $\mathbb{R}^{4}$ tangent to the sphere $\mathbb{S}^{3}$.

Now we can extend analytically the vector field $\bar{X}(y)$ to the whole sphere $\mathbb{S}^{3}$ by

$$
p(X)(y)=y_{4}^{n-1} \bar{X}(y) ;
$$

this extended vector field $p(X)$ is called the Poincaré compactification of $X$.

As $\mathbb{S}^{3}$ is a differentiable manifold, to compute the expression for $p(X)$ we can consider the eight local charts $\left(U_{i}, F_{i}\right),\left(V_{i}, G_{i}\right)$ where $U_{i}=\left\{y \in \mathbb{S}^{3}: y_{i}>0\right\}$, and $V_{i}=\left\{y \in \mathbb{S}^{3}: y_{i}<0\right\}$ for $i=1,2,3,4$; the diffeomorphisms $F_{i}: U_{i} \rightarrow \mathbb{R}^{3}$ and $G_{i}: V_{i} \rightarrow \mathbb{R}^{3}$ for $i=1,2,3,4$ are the inverses of the central projections from the origin to the tangent planes at the points $( \pm 1,0,0,0),(0, \pm 1,0,0),(0,0, \pm 1,0)$ and $(0,0,0, \pm 1)$, respectively. We now do the computations on $U_{1}$. Suppose that the origin $(0,0,0,0)$, the point $\left(y_{1}, y_{2}, y_{3}, y_{4}\right) \in \mathbb{S}^{3}$ and the point $\left(1, z_{1}, z_{2}, z_{3}\right)$ in the tangent plane to $\mathbb{S}^{3}$ at $(1,0,0,0)$ are collinear, then we have

$$
\frac{1}{y_{1}}=\frac{z_{1}}{y_{2}}=\frac{z_{2}}{y_{3}}=\frac{z_{3}}{y_{4}}
$$

and consequently

$$
F_{1}(y)=\left(\frac{y_{2}}{y_{1}}, \frac{y_{3}}{y_{1}}, \frac{y_{4}}{y_{1}}\right)=\left(z_{1}, z_{2}, z_{3}\right)
$$

defines the coordinates on $U_{1}$.

As

$$
D F_{1}(y)=\left(\begin{array}{cccc}
-y_{2} / y_{1}^{2} & 1 / y_{1} & 0 & 0 \\
-y_{3} / y_{1}^{2} & 0 & 1 / y_{1} & 0 \\
-y_{4} / y_{1}^{2} & 0 & 0 & 1 / y_{1}
\end{array}\right)
$$


and $y_{4}^{n-1}=\left(\frac{z_{3}}{\Delta z}\right)^{n-1}$, the analytical field $p(X)$ in $U_{1}$ becomes

$$
\frac{z_{3}^{n}}{(\Delta z)^{n-1}}\left(-z_{1} P^{1}+P^{2},-z_{2} P^{1}+P^{3},-z_{3} P^{1}\right),
$$

where $P^{i}=P^{i}\left(1 / z_{3}, z_{1} / z_{3}, z_{2} / z_{3}\right)$.

In a similar way we can deduce the expressions of $p(X)$ in $U_{2}$ and $U_{3}$. These are

$$
\frac{z_{3}^{n}}{(\Delta z)^{n-1}}\left(-z_{1} P^{2}+P^{1},-z_{2} P^{2}+P^{3},-z_{3} P^{2}\right),
$$

where $P^{i}=P^{i}\left(z_{1} / z_{3}, 1 / z_{3}, z_{2} / z_{3}\right)$ in $U_{2}$, and

$$
\frac{z_{3}^{n}}{(\Delta z)^{n-1}}\left(-z_{1} P^{3}+P^{1},-z_{2} P^{3}+P^{2},-z_{3} P^{3}\right),
$$

where $P^{i}=P^{i}\left(z_{1} / z_{3}, z_{2} / z_{3}, 1 / z_{3}\right)$ in $U_{3}$.

The expression for $p(X)$ in $U_{4}$ is $z_{3}^{n+1}\left(P^{1}, P^{2}, P^{3}\right)$ where the component $P^{i}=P^{i}\left(z_{1}, z_{2}, z_{3}\right)$.

The expression for $p(X)$ in the local chart $V_{i}$ is the same as in $U_{i}$ multiplied by $(-1)^{n-1}$.

When we shall work with the expression of the compactified vector field $p(X)$ in the local charts we omit the factor $1 /(\Delta z)^{n-1}$. We can do that through a rescaling of the time.

We remark that all the points on the sphere at infinity in the coordinates of any local chart have $z_{3}=0$.

The ortogonal projection of the closed north hemisphere of $\mathbb{S}^{3}$ on the hyperplane $y_{4}=0$ is a closed ball $B$ of radius 1 centered at the origin of coordinates, whose interior is diffeomorphic to $\mathbb{R}^{3}=T_{(0,0,0,1)} \mathbb{S}^{3}$ and whose boundary $\mathbb{S}^{2}$ is the infinity of $\mathbb{R}^{3}$. This closed ball $B$ is the Poincaré ball.

\section{ACKNOWLEDGMENTS}

The first author is partially supported by the grants MICINN/FEDER MTM 2008-03437, AGAUR 2009SGR 410 and ICREA Academia. The second author is partially supported by Dirección de Investigación DIUBB 1204084/R.

\section{REFERENCES}

[1] B. Abraham-Shrauner, P. Leach, K. Govinder and G. Ratcliff, Hidden and contact symmetries of ordinary differential equations, J. Phys. A: Math. Gen. 28, (1995), 6707-6716. 
[2] A. Cima And J. Llibre, Bounded polynomial vector fields, Trans. Amer. Math. Soc. 318 (1990), 557-579.

[3] F. Dumortier, J. Llibre And J.C. Artés, Qualitative theory of planar differential systems, UniversiText, Springer-Verlag, New York, 2006.

[4] K. Goviender AND P. LEACH, The algebraic structure of the first integrals of third-order linear equations, J. Math. Anal. Appl. 195 (1995), 114-133.

[5] P. LEACH, On the uniqueness of the Schwarzian and linearisation by nonlocal contact transformation, J. Math. Anal. Appl. 235 (1999), 84-107.

[6] P. LEACH, Symmetry and singularity properties of the generalised KummerSchwarz and related topics, J. Math. Anal. Appl. 348 (2008), 487-493.

[7] C. Vidal And P. Gómez, An extension of the Poincaré compactification and a geometric interpretation, Proyecciones 22, 3 (2003), 161-180.

${ }^{1}$ Departament de Matemàtiques, Universitat Autònoma de Barcelona, 08193 Bellaterra, Barcelona, Catalonia, Spain

E-mail address: jllibre@mat.uab.cat

2 Departamento de Matemática, Universidad del Bio Bio, Concepción, Avda. Collao 1202, Chile

E-mail address: clvidal@ubiobio.cl 